\title{
Efecto de Tres Tipos de Empadre y Dos Tipos de Alimentación sobre los Índices Reproductivos en Cuyes Criados en la Sierra Peruana
}

\author{
Effect of Three Mating Types and Two Feeding Types on Reproductive \\ Parameters in Guinea Pig Reared in the Peruvian Highlands \\ Silvia Velásquez C. ${ }^{1}$, Ronald Jiménez A. ${ }^{1,3}$, Amparo Huamán C. ${ }^{1}$, \\ Felipe San Martín H. ${ }^{2}$, Fernando Carcelén C. ${ }^{2}$
}

\section{Resumen}

En este estudio se evaluó el efecto de tres tipos de empadre y dos tipos de alimentación sobre parámetros reproductivos. Se utilizaron 210 cuyes hembras y 30 machos reproductores en un diseño de bloques completos al azar con arreglo factorial de tres tipos de empadre (posparto, posdestete y posdescanso) x dos tipos de alimentación (forraje verde, forraje verde más suplemento). El estudio comprendió la evaluación de las reproductoras durante el segundo y tercer parto. Se encontró efecto del tipo de empadre sobre las variables peso al nacimiento, número de partos por año y uso eficiente del celo $(\mathrm{p}<0.05)$. También hubo efecto del tipo de alimentación sobre la variable peso al nacimiento $(\mathrm{p}<0.05)$. La interacción tipo de alimento por tipo de empadre fue significativa, indicando que la suplementación tiende a mejorar la tasa de fertilidad, el tamaño de camada y el índice de productividad anual en el empadre posparto y tiende a reducirlo en los empadres posdestete y posdescanso proporcionalmente al tiempo trascurrido desde el parto al inicio del empadre $(p<0.05)$. En crianzas de cuyes en la sierra peruana se recomienda el empadre posparto con suplementación energética en lugar de una ración de forraje verde a discreción o el empadre posdestete con alimentación de forraje verde a discreción.

Palabras clave: cuyes, empadre, alimentación, reproducción, productividad

${ }^{1}$ Estación Experimental El Mantaro, Centro de Investigación IVITA, Facultad de Medicina Veterinaria, Universidad Nacional Mayor de San Marcos, Jauja, Perú

${ }^{2}$ Laboratorio de Bioquímica, Nutrición y Alimentación Animal, Facultad de Medicina Veterinaria, Universidad Nacional Mayor de San Marcos, Lima, Perú

${ }^{3}$ E-mail:rjimeneza@unmsm.edu.pe 
This study aimed to evaluate the effect of three mating types and two feeding types on reproductive parameters. A total of 210 females and 30 males guinea pigs were used in a complete block design with a factorial arrangement of three mating types (post-partum, post weaning, 7-day after weaning) $\mathrm{x}$ two feeding types (green forage, green forage with supplement). The study included the evaluation of the reproductive performance of females between the second and third parturition. The results showed the significant effect of the mating type on birth weight, number of parturitions per year and the efficient use of estrus $(p<0.05)$. Additionally, there was a significant effect of feeding type on birth weight $(\mathrm{p}<0.05)$. The interaction feeding and mating type was significant indicating that supplementation tends to improve fertility rate, litter size and annual productivity index when applying the post-partum mating; likewise, it tends to proportionally reduce them according to the interval between parturition and the beginning of mating in the postweaning and 7-day after weaning mating types $(\mathrm{p}<0.05)$. In guinea pig breeding in the Peruvian highlands is recommended the post-partum mating with energy supplementation instead of only green forage or post-weaning mating using green forage.

Key words: guinea pig, mating, feeding, reproduction, productivity

\section{INTRODUCCIÓN}

Los cuyes están fisiológicamente dotados para mantener una reproducción acelerada, legado de sus ancestros silvestres, pues para ellos la reproducción contribuía a la sobrevivencia de la especie ante las pérdidas producidas por depredación. Entre las características reproductivas se señala que alcanzan la pubertad a partir de los 45 días, el ciclo estrual es de 16 días, la gestación de 68 días, el tamaño de camada de 1 a 8 y el $70 \%$ de las hembras presenta celo a pocas horas del parto (Aliaga et al., 2009).

El inicio de la pubertad en las hembras es dependiente del peso vivo y se alcanza entre los 45 a 60 días (Aliaga et al., 2009). No obstante esta precocidad, se prefiere iniciar la etapa reproductiva cuando las hembras alcanzan el $60 \%$ del peso vivo adulto, el cual oscila entre 800 y $900 \mathrm{~g}$ en cuyes mejorados (Jiménez y Huamán, 2010), a fin de obtener una mejor respuesta en tamaño y peso de camada (Chauca, 1997). Por otro lado, pesos mayores a 1000 g o edades mayores a 3 meses en hembras primerizas puede ocasionar partos distócicos (Aliaga et al., 2009).

Es importante que los machos sean de mayor peso y tamaño que las hembras pues esta característica favorece la dominancia y facilita la cópula (Jiménez y Huamán, 2010).

Los sistemas de empadre se diferencian por el tipo de celo que se aprovecha. En el empadre continuo se mantiene el macho con las hembras, de modo que se aprovecha el celo posparto (Chauca 1997; Aliaga et al., 2009). Esto permite un mayor número de partos por año; sin embargo, la alta exigencia puede afectar la productividad debido a una mayor mortalidad de crías. Por otro lado, el ingreso de los machos, en algunos casos en días posteriores al parto y en otros hasta después del destete, favorece la obtención de crías más vigorosas, homogéneas y con mayor probabilidad de supervivencia (Bustamante, 1993). 
Técnicamente, Aliaga et al. (2009) propone hasta siete tipos de empadre, dependiendo del celo empleado. Chauca (1997) señala que los tipos de empadre se diferencian entre los que aprovechan el celo postparto y los que no lo hacen. En términos prácticos, las crianzas comerciales de cuyes del valle del Mantaro practican tanto el empadre continuo como el empadre posdestete o el empadre posdescanso ( $\mathrm{R}$ Jiménez, Jauja, comunicación personal).

El éxito del manejo reproductivo se da cuando hay correspondencia con una adecuada alimentación. El cuy, por ser un herbívoro fermentador posgástrico, aprovecha adecuadamente el forraje verde que cubre los requerimientos de vitaminas, minerales y agua (Rico et al., 1994). Sin embargo, en un sistema de mayor exigencia productiva, como el de una crianza comercial, el forraje verde tiene limitaciones para cubrir las demandas energéticas de los cuyes, de allí que la suplementación de energía se hace necesaria (Jiménez y Huamán, 2010). El aporte energético no supera a 2.5 Mcal de energía digestible (ED) por kilo de materia seca (MS) cuando la alimentación es solo con pasturas (Laforé et al., 1999), de modo que no llega a satisfacer las necesidades de los cuyes, estimado en $2.8 \mathrm{Mcal} \mathrm{ED} / \mathrm{kg}$ MS (Zaldívar et al., 1988). No obstante, el cuy recurre a altas tasas de consumo, que llegan hasta el 10\% de su peso vivo en MS, para satisfacer sus necesidades energéticas cuando son alimentados con pastos cultivados (Jiménez y Huamán, 2010).

En el valle del Mantaro se dispone de subproductos agrícolas variados (Laforé et al., 1999) que se utilizan como suplemento en la alimentación de cuyes y que aportan importantes cantidades de energía y proteína; entre estos, la cebada, la avena y el trigo (Jiménez, 2007). La cebada es frecuentemente utilizada como suplemento por su alto contenido energético, que se encuentra alrededor de $3.68 \mathrm{Mcal} \mathrm{ED} / \mathrm{kg}$ MS (Laforé et al., 1999).
Se conoce que una suplementación energética y proteica en especies como la caprina, ovina y cuy, por encima de sus necesidades en la etapa previa y durante el empadre, resulta en un mayor número de crías (Forcada et al., 1992; Acero 2007; Aliaga et al., 2009). Además, Martínez de Acurero et al., (1986) y Martínez de Acurero (2000), concluyen que la suplementación energética en el ovino es de mayor importancia que la proteica en la etapa anterior al empadre para lograr un efecto estimulador en la formación de folículos preovulatorios.

En la actualidad no existe un manejo alimenticio ni reproductivo que demuestre ser el más apropiado desde el punto de vista productivo para crianzas comerciales de cuyes en Sierra. Es así que el presente estudio evaluó los empadres de tipo continuo, posdestete y posdescanso en combinación con dos tipos de alimentación diferenciados, uno a base de forraje verde y otro a base de forraje verde suplementado con harina de cebada, sobre los parámetros reproductivos en cuyes criados en la sierra peruana.

\section{Materiales y Métodos}

\section{Lugar de Ejecución}

El estudio se realizó entre enero y diciembre de 2008 en la Unidad de Investigación en Cuyes de la Estación El Mantaro del Centro de Investigaciones IVITA, ubicada en el km 34 de la carretera Huancayo-Jauja, provincia de Jauja, Junín, Perú, a una altitud de $3320 \mathrm{msnm}$. La zona presenta una temperatura ambiental que oscila entre 19.9 y $-1.8^{\circ} \mathrm{C}$ y una precipitación pluvial promedio anual de $770 \mathrm{~mm}$

\section{Animales Experimentales}

Se emplearon 210 cuyes hembras de primer empadre y 30 machos reproductores, de tipo mejorados por selección en ganancia de peso. 


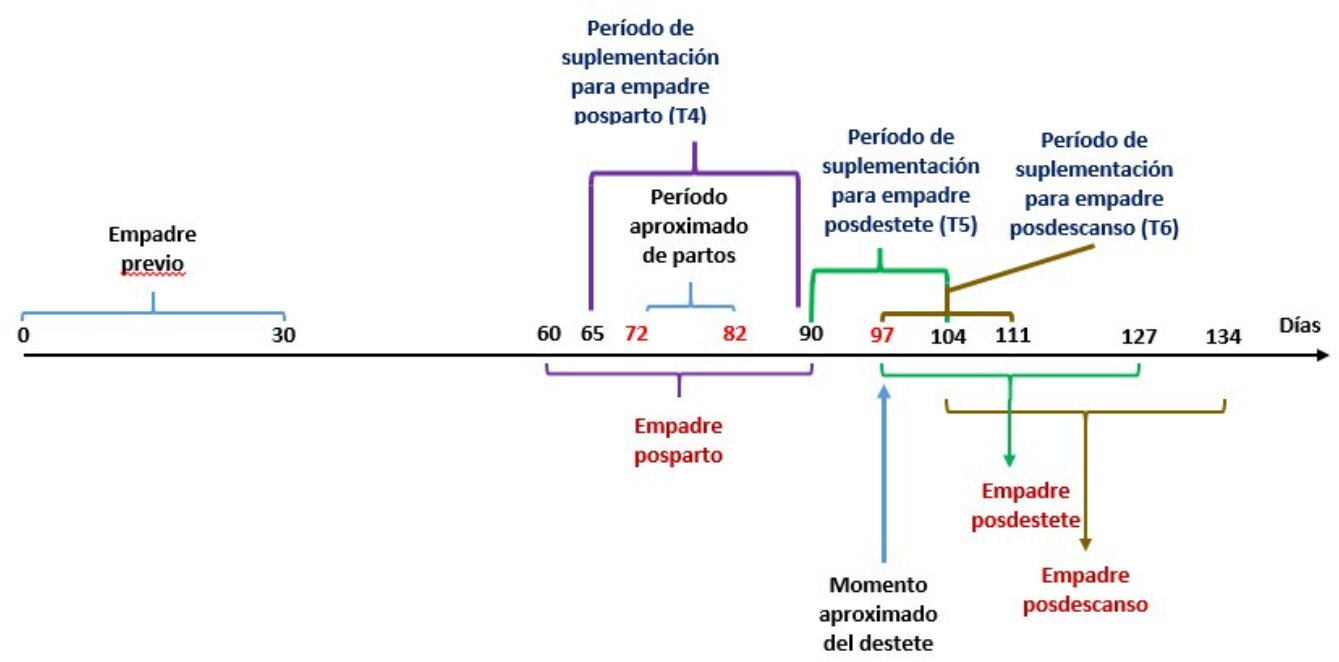

Figura 1. Periodos de suplementación con harina de cebada en los tratamientos T4, T5 y T6

Hembras de $600 \mathrm{~g}$ fueron agrupadas aleatoriamente en núcleos reproductivos de siete animales. Al alcanzar $800 \mathrm{~g}$ de peso se inició el empadre introduciendo un macho de $1200 \mathrm{~g}$ de peso y fertilidad probada en cada núcleo reproductivo. Para ello, el macho estuvo previamente con dos hembras jóvenes por 20 días y luego de diagnosticar la gestación positiva de las hembras se le catalogó como fértil. Este primer empadre duró 30 días.

\section{Manejo Experimental}

Los núcleos reproductivos constituidos fueron alojados en pozas de $1.5 \times 1.5 \mathrm{~m}$ de área y $0.5 \mathrm{~m}$ de altura, construidas a base de madera de eucalipto y malla metálica cuadrada de una pulgada. Los machos durante el periodo que no empadraban estuvieron en pozas individuales de $0.8 \times 0.8 \mathrm{~m}$ de área y $0.5 \mathrm{~m}$ de altura, de materiales similares. Los cuyes tuvieron el espacio vital recomendado por Cáceres et al. (2004).

Previo al ingreso de los animales, las pozas se dotaron de cama de $5 \mathrm{~cm}$ de espesor a base de paja de cebada. Desde el inicio de los núcleos reproductivos y hasta antes de iniciar los tratamientos, los animales fueron alimentados con forraje verde, compuesto por pastura asociada (alfalfa variedad california 55 , ryegrass italiano de la variedad Belinda y trébol rojo variedad Quinequelli), en cantidades de $500 \mathrm{~g}$ de forraje verde por cuy/día cuando las hembras tuvieron $600 \mathrm{~g}$ de peso. Esta cantidad fue incrementándose en $50 \mathrm{~g}$ adicionales por cada semana hasta llegar a $700 \mathrm{~g}$ de forraje verde por cuy/día; periodo en que las hembras alcanzaron $800 \mathrm{~g}$ de peso vivo.

Desde el inicio del empadre hasta el inicio de los tratamientos, los cuyes se alimentaron con el mismo forraje verde asociado ofrecido a discreción, lo cual garantizaba una cantidad equivalente a $750 \mathrm{~g}$ de forraje verde por cuy/día.

\section{Tratamientos}

\section{Tipos de empadre}

- Empadre posparto (EPP). El macho ingresa 30 días después de finalizado el primer empadre (empadre previo); momento en que las hembras se encuentran próximas al parto.

- Empadre posdestete (EPD). El macho ingresa el día del destete (15 días después del último parto). 
- Empadre posdescanso (EPDO). El macho ingresa siete días después del destete.

En todos los casos, el macho permanece con las hembras por 30 días.

\section{Tipos de alimentación}

- Forraje verde (FV). Forraje verde a discreción (se ofreció $800 \mathrm{~g}$ de la pastura asociada por cuy/día; cantidad que permitió dejar residuos)

- Forraje verde más harina de cebada $(\mathrm{FV}+\mathrm{HC})$. Además del FV a discreción recibe suplementación con harina de cebada (HC).

Se trabajó con seis tratamientos, producto de la combinación de tipos de empadre y tipo de alimentación:

- T1: EPP y FV

- T2: EPD y FV

- T3: EPDO y FV

- T4: EPP y FV+HC. Suplementación de $30 \mathrm{~g}$ de $\mathrm{HC}$ por cuy/día desde 7 días previos al inicio del empadre hasta 7 días posteriores al último parto (Figura 1).

- T5: EPD y FV+HC. Suplementación de $30 \mathrm{~g}$ de $\mathrm{HC}$ por cuy/día desde 7 días previos al inicio del empadre y por 14 días (Figura 1).

- T6: EPDO y FV+HC. Suplementación de $30 \mathrm{~g}$ de $\mathrm{HC}$ por cuy/día desde el destete y por 14 días (Figura 1).

\section{Variables}

- Tasa de fertilidad: Proporción de hembras preñadas respecto a hembras empadradas.

- Tamaño de camada: Total de crías nacidas entre total de hembras paridas.

- Sobrevivencia al nacimiento: Proporción de crías nacidas vivas respecto a crías totales.

- Sobrevivencia al destete: Proporción de crías logradas al destete respecto a crías nacidas vivas.

- Peso promedio al nacimiento: Peso de la camada entre el tamaño de camada.
- Número de partos por año: Resulta de dividir 365 entre el intervalo promedio parto-parto.

- Productividad acumulada de segundo y tercer parto: Expresa la cantidad de crías destetadas obtenidas por reproductora en el segundo y tercer parto.

- Índice de productividad anual estima$d o$ : Resulta de multiplicar los valores de tasa de fertilidad, tamaño de camada, sobrevivencia al nacimiento, sobrevivencia al destete y número de partos por año. Estima la cantidad de crías destetadas que produce una hembra reproductora por año.

- Uso eficiente del celo: Proporción de celos aprovechados respecto a celos presentados (asumiendo que después del celo posparto, que se presenta a 3-5 horas del parto, los siguientes celos se presentaron cada 16 días [Aliaga et al., 2009]).

\section{Diseño Experimental}

Se empleó un diseño de bloques completos al azar con arreglo factorial de 2 (tipo de alimentación) x 3 (tipo de empadre), con cinco unidades experimentales por tratamiento y cada unidad experimental constituida por un núcleo de siete reproductoras de primer empadre, las cuales se evaluaron entre el segundo y tercer empadre.

\section{Análisis de Datos}

Los datos fueron analizados mediante análisis de varianza empleando el paquete estadístico SAS 9.1 (SAS, 1990).

\section{Resultados y Discusión}

La tasa de fertilidad, tamaño de camada e índice de productividad anual estimado fueron afectados por la interacción tipo de empadre $\mathrm{x}$ tipo de alimentación (Cuadro 1; Figura 2a,b,c). En los tipos de empadre donde no se aprovecha el celo post parto se 
Cuadro 1. Efecto del tipo de alimentación y tipo de empadre sobre parámetros reproductivos en cuyes

\begin{tabular}{|c|c|c|c|c|c|c|c|}
\hline & \multicolumn{3}{|c|}{ Forraje verde } & \multicolumn{3}{|c|}{$\begin{array}{l}\text { Forraje verde }+ \text { harina de } \\
\text { cebada }\end{array}$} & \multirow{2}{*}{$\mathrm{CME}^{4}$} \\
\hline & $\begin{array}{l}\text { Pos- } \\
\text { parto }\end{array}$ & $\begin{array}{c}\text { Pos- } \\
\text { destete }\end{array}$ & $\begin{array}{c}\text { Pos- } \\
\text { descanso }\end{array}$ & $\begin{array}{l}\text { Pos- } \\
\text { parto }\end{array}$ & $\begin{array}{l}\text { Pos- } \\
\text { destete }\end{array}$ & $\begin{array}{c}\text { Pos- } \\
\text { descanso }\end{array}$ & \\
\hline Fertilidad $(\%)^{3}$ & 66 & 93 & 90 & 74 & 87 & 59 & 0.03 \\
\hline $\begin{array}{l}\text { Tamaño de } \\
\text { camada }^{3}\end{array}$ & 2.5 & 3.6 & 3.8 & 3.4 & 2.9 & 3.4 & 0.42 \\
\hline $\begin{array}{l}\text { Sobrevivencia al } \\
\text { nacimiento }(\%)\end{array}$ & 92 & 83 & 77 & 83 & 84 & 92 & 0.05 \\
\hline $\begin{array}{l}\text { Sobrevivencia al } \\
\text { destete }(\%)\end{array}$ & 94 & 95 & 90 & 96 & 82 & 92 & 0.03 \\
\hline $\begin{array}{l}\text { Peso promedio al } \\
\text { nacimiento }(\mathrm{g})^{1}\end{array}$ & 148 & 137 & 139 & 141 & 149 & 120 & 199 \\
\hline $\begin{array}{l}\text { Número de partos } \\
\text { por año }{ }^{1,2}\end{array}$ & 3.6 & 3.6 & 3.3 & 4.5 & 3.7 & 3.1 & 0.12 \\
\hline $\begin{array}{l}\text { Productividad } \\
\text { acumulada de } 2^{\circ} \\
\text { y } 3^{\circ} \text { parto }\end{array}$ & 3.2 & 5.0 & 4.5 & 4.0 & 3.6 & 3.7 & 1.4 \\
\hline $\begin{array}{l}\text { Índice }{ }^{6} \text { de } \\
\text { productividad } \\
\text { anual }^{3}\end{array}$ & 6.0 & 9.0 & 7.5 & 9.4 & 6.6 & 6.3 & 6.6 \\
\hline $\begin{array}{l}\text { Uso eficiente del } \\
\text { celo }(\%)^{1}\end{array}$ & 39 & 16 & 57 & 63 & 23 & 56 & 0.07 \\
\hline
\end{tabular}

${ }^{1}$ Variables de respuesta que tienen efecto $(p<0.05)$ del tipo de empadre

${ }^{2}$ Variables de respuesta que tienen efecto $(p<0.05)$ del tipo de alimentación

${ }^{3}$ Variables de respuesta que tienen efecto $(p<0.05)$ de la interacción tipo de empadre por tipo de alimentación

${ }^{4}$ Cuadrado medio del error

${ }^{5}$ Crías destetadas /reproductora $/ 2^{\circ}$ y $3^{\text {er }}$ parto

${ }^{6}$ Crías destetadas/reproductora/año

observó una disminución en la tasa de fertilidad, tamaño de camada e índice de productividad anual estimado cuando los cuyes fueron alimentados con forraje verde y suplementados con harina de cebada en relación al empadre posparto, donde estos parámetros fueron superiores.

Cuando los cuyes se alimentan con solo forraje verde ocurre un marcado balance energético negativo posparto, dado que el aporte energético de los pastos es insuficiente para cubrir las necesidades de lactación y reproducción (Jiménez y Huamán, 2010), lo que determina una baja respuesta en fertilidad, tamaño de camada, productividad acumulada de $2^{\circ}$ y $3^{\circ}$ parto e índice de productividad anual estimado en las hembras con empadre posparto. La respuesta mejora cuando se suplementa con harina de cebada (Cuadro 1, Figura 2a,b,c), debido un posible incremento de las reservas de tejido adiposo, 

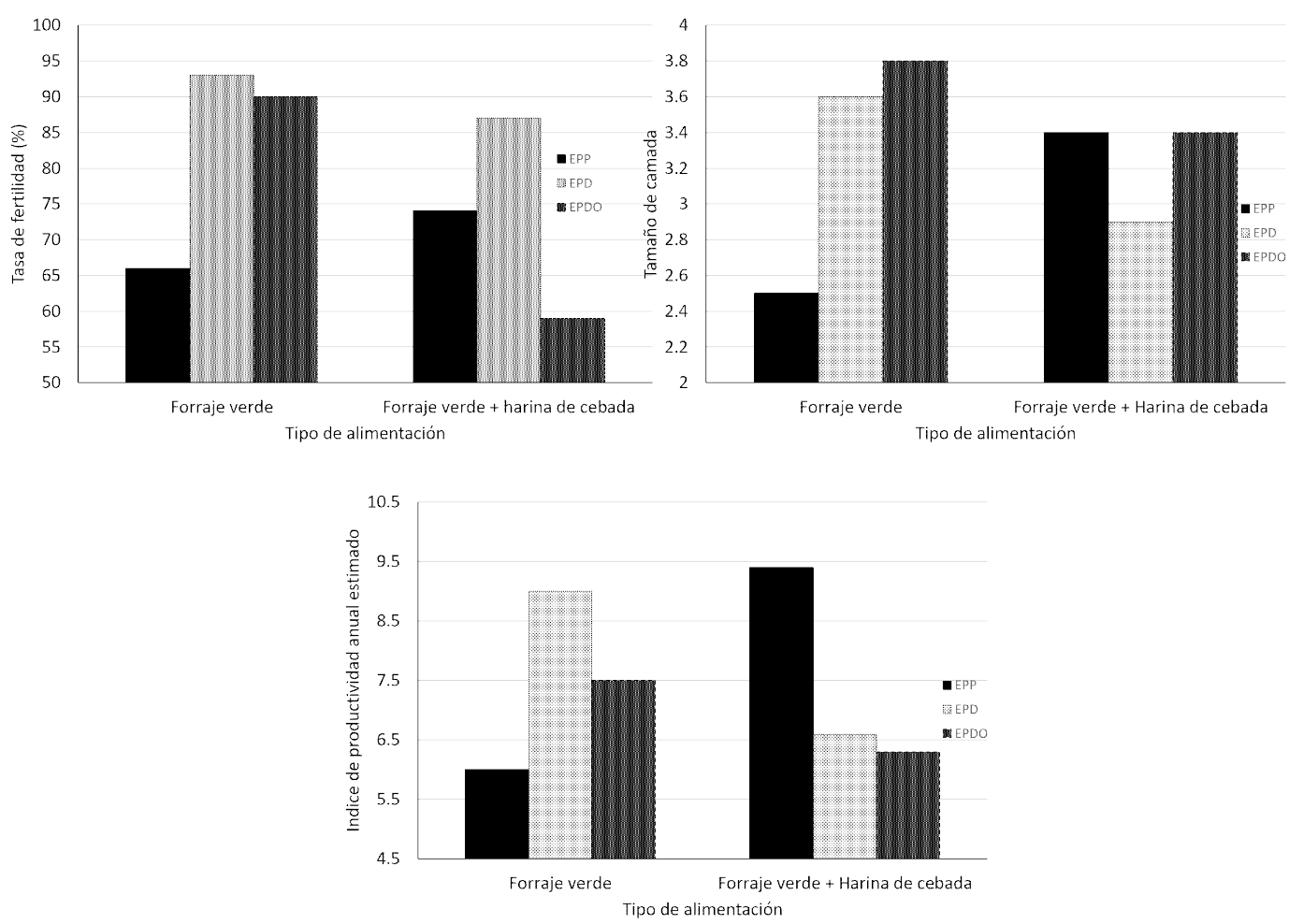

Figura 2. Efecto de la interacción tipo de empadre y tipo de alimentación sobre: a) la tasa de fertilidad en cuyes, b) tamaño de camada, c) índice de productividad anual estimado en cuyes

mejorando el índice de condición corporal (ICC) (Ara et al., 2012). Niveles de ICC cercanos a tres al inicio del empadre favorecen la respuesta reproductiva de los cuyes (Jiménez y Huamán, 2010), porque el balance energético es óptimo y donde hormonas como la leptina promueven la fertilidad (Smith et al., 2002) y la gestación exitosa (PérezPérez et al., 2015). La suplementación energética en momentos de desbalance energético también mejora la fertilidad en vacas (Aguilar-Pérez et al., 2009), borregas (Hashem y El-Zarkouny, 2013) y conejas (Church et al., 2010).

En el caso de cuyes alimentados con solo forraje verde, en contraste a lo que ocurre en el empadre posparto, las hembras en el empadre posdestete dejaron de requerir nutrientes para lactación y en el empadre posdescanso tuvieron una semana sin lactación antes de iniciar el empadre. Bajo estas circunstancias, el balance energético en el empadre posdestete llega a equilibrarse con tendencia a ser positivo y es positivo durante el empadre posdescanso. En este argumento se basa Jiménez y Huamán (2010) para proponer alimentar con forraje verde en el empadre posdescanso y forraje verde con suplementación energética en el empadre posdestete a fin de lograr una adecuada respuesta reproductiva.

La suplementación energética en los empadres posdestete y posdescanso incrementaron adicionalmente el balance energético positivo $\mathrm{y}$, consecuentemente, las reservas de tejido adiposo y el ICC (Ara et al., 2012). No obstante, se encontró una menor respuesta reproductiva respecto a la ali- 
Cuadro 2. Efecto del tipo de empadre sobre parámetros reproductivos en cuyes

\begin{tabular}{lccc}
\hline Empadre & $\begin{array}{c}\text { Empadre } \\
\text { posparto }\end{array}$ & $\begin{array}{l}\text { Empadre pos- } \\
\text { destete }\end{array}$ & $\begin{array}{c}\text { Empadre pos- } \\
\text { descanso }\end{array}$ \\
\hline Fertilidad (\%) & 70 & 88 & 74 \\
Tamaño de camada & 3.0 & 3.3 & 3.6 \\
Sobrevivencia al nacimiento (\%) & 85 & 83 & 80 \\
Sobrevivencia al destete (\%) & 90 & 88 & 87 \\
Peso promedio al nacimiento (g) & $144.4^{\mathrm{a}}$ & $141.6^{\mathrm{ab}}$ & $128.6^{\mathrm{b}}$ \\
Número de partos por año & $4.2^{\mathrm{a}}$ & $3.7^{\mathrm{b}}$ & $3.4^{\mathrm{b}}$ \\
$\begin{array}{l}\text { Productividad acumulada de } 2^{\circ} \text { y } 3^{\text {er }} \\
\text { parto al destete }\end{array}$ & 3.6 & 4.3 & 4.1 \\
Índice de productividad anual estimada & 7.71 & 7.83 & 6.90 \\
(crías/reproductora/año) & & & \\
Uso eficiente del celo (\%) & $52^{\mathrm{a}}$ & $21^{\mathrm{b}}$ & $56^{\mathrm{a}}$ \\
\hline
\end{tabular}

$a, b$ Letras diferentes dentro de filas señalan diferencias significativas $(p<0.05)$

Cuadro 3. Efecto del tipo de alimentación sobre parámetros reproductivos en cuyes

\begin{tabular}{lcc}
\hline Alimentación & Forraje verde & $\begin{array}{c}\text { Forraje verde }+ \\
\text { harina de cebada }\end{array}$ \\
\hline Fertilidad (\%) & 81 & 73 \\
Tamaño de camada & 3.3 & 3.2 \\
Sobrevivencia al nacimiento (\%) & 82 & 83 \\
Sobrevivencia al destete (\%) & 89 & 87 \\
Peso promedio al nacimiento (g) & 141.1 & 135.3 \\
Número de partos por año & $3.6^{\mathrm{b}}$ & $3.9^{\mathrm{a}}$ \\
Productividad acumulada de $2^{\mathrm{o}}$ y $3^{\text {er }}$ parto & 4.3 & 3.8 \\
al destete & & 7.4 \\
Índice de productividad anual estimada & 7.5 & 48 \\
(crías/reproductora/año) & 38 & \\
Uso eficiente del celo (\%) & & \\
\hline
\end{tabular}

${ }^{a, b}$ Letras diferentes dentro de filas señalan diferencias significativas $(p<0.05)$

mentación con solo forraje verde (Cuadro 1, Figura 2a,b,c). Al respecto, Michael y Bonnet (2011) hallaron una relación entre la acumulación excesiva de tejido adiposo y la inhibición reproductiva, atribuido al efecto supre- sor de la esteroidogénesis en el ovario, generado por altos niveles de leptina. Cuando la leptina se encuentra elevada favorece la reproducción, pero niveles deficientes o muy altos son perjudiciales para la ovulación (Smith 
et al., 2002; Pérez-Pérez et al., 2015) y probablemente para la implantación embrionaria (Brewer y Balen, 2010; Barrios de Tomasi et al., 2013). Estos argumentos explican la baja respuesta reproductiva en los empadres posdestete y posdescanso con suplementación, destacándose la disminución en $31 \%$ en la tasa de fertilidad en el empadre posdescanso (Cuadro 1).

Las diferencias en tasas de fertilidad entre los tipos de empadre son marcadas, pero no llegan a ser significativas (Cuadro 1). El empadre posdestete obtuvo el mayor valor en la tasa de fertilidad ( $88 \%$ ) y la menor variación por efecto del tipo de alimentación (Cuadros 2 y 3). Es decir, no hubo mayor ventaja de suplementar energéticamente reproductoras que se manejan con empadre posdestete.

Aliaga et al. (1984) señalan que 74 a $80 \%$ de cuyes hembras paridas presentan celo posparto y aproximadamente el $70 \%$ llega a preñar; datos que permiten determinar que la tasa de fertilidad en el empadre posparto oscila entre 52 a $56 \%$. Estos valores fueron superados en el presente estudio (66-74\%; Cuadros 1 y 2).

De las investigaciones realizadas por Aliaga et al. (2009) y lo observado en el presente estudio, se infiere que el empadre posparto responde favorablemente en fertilidad y tamaño de camada con suplementaciones parciales o permanentes, mientras que el empadre controlado puede experimentar una respuesta variable a la suplementación en estas mismas variables.

El 77 a $92 \%$ de sobrevivencia al nacimiento fueron cercanos a los valores de 88$96 \%$ reportados por Aliaga et al. (1984), mientras que los valores de 82 a $96 \%$ de sobrevivencia al destete fueron superiores al $60-83 \%$ reportado por este autor.

El peso promedio al nacimiento estuvo afectado por el tipo de empadre (Cuadros 1 y 2). El peso de las crías obtenidas con el empadre posparto fue superior al peso de las crías logradas con el empadre posdescanso $(p<0.05)$ y similar al obtenido con el empadre posdestete. Estos datos son inusuales dado que la tendencia es que los menores pesos al nacimiento se observen en el empadre posparto (Bustamante, 1993; Aliaga et al., 2009).

El empadre posparto obtuvo el mayor número de partos por año (Cuadro 2), pero menor al reportado por Chauca (1997), lo que da a entender que no todas las hembras presentaron celos posparto y no todos los primeros celos posparto fueron aprovechados. Asimismo, la suplementación con harina de cebada permitió un mayor número de partos por año (Cuadro 2), probablemente debido a que al mejorar el ICC de las hembras, las fertilizaciones son más efectivas y, por lo tanto, se acortan los intervalos parto-parto.

Los resultados de productividad acumulada de segundo y tercer parto se encuentran entre 3.2 a 5.0 crías destetadas por reproductora, en tanto que el índice de productividad estimado fue de 6.3 y 9.4 crías destetadas por reproductora/año. Según los resultados del presente estudio, se puede emplear alternativamente el empadre posdestete con alimentación a base de forraje verde a discreción (T2) o el empadre posparto con alimentación de forraje verde suplementado con harina de cebada alrededor del periodo de empadre (T4). En ambos casos se obtiene un índice mayor a 9, el cual es satisfactorio para la sierra central peruana, dado que el promedio se encuentra en 7.26; no obstante, los cuyes reproductores $\mathrm{G}$, cuyos machos son producto del cruzamiento de las líneas Precoz y Cárnica y las hembras cruce de las líneas Prolífica y Lechera, alcanzan valores de 12 (Jiménez y Huamán, 2010).

Aliaga et al. (1984) encontraron que la suplementación permanente con empadre posparto maximiza el índice de productividad (10.44), pero disminuye con el empadre controlado (7.87); sin embargo, el empadre 
controlado obtiene mejor productividad con suplementación en momentos previos y a inicios del empadre (10.0). El comportamiento de estos resultados se asemeja al presente estudio donde se observa que si el aporte de energía del suplemento supera los requerimientos de la hembra se producen efectos negativos en la reproducción.

El uso eficiente del celo es relativamente bajo en los tres tipos de empadre (16-63\%) y tiene influencia del tipo de empadre $(\mathrm{p}<0.05)$, donde el uso eficiente del celo es menor en el empadre posdestete $(21 \%)$ respecto al empadre posdescanso (56\%) y posparto (52\%) (Cuadro 2). También es importante destacar que el empadre posparto mejoró en $18 \%$ el uso eficiente del celo cuando se dio la suplementación energética alrededor del empadre (Cuadro 1).

Los empadres posparto y posdescanso permitieron una mejor utilización de los celos previstos. En cambio, en el posdestete, donde el inicio del empadre estuvo determinado por la edad de las últimas crías (15 días), y donde muchas hembras ya tuvieron su segundo celo posparto, no pudiendo ser fertilizadas sino hasta el tercer celo posparto.

\section{Conclusiones}

- La suplementación con harina de cebada desde una semana antes del inicio del empadre y durante dos semanas mejora la fertilidad, tamaño de camada e índice de productividad anual cuando se emplea el tipo de empadre posparto, pero lo afecta negativamente cuanto más tardío sea el inicio del empadre respecto a los partos.

- Las combinaciones de empadre posdestete con alimentación forrajera y el empadre posparto con suplementación energética presentaron el mayor índice de productividad anual estimado.

\section{Literatura Citada}

1. Acero R. 2007. Evaluación de dos estrategias de alimentación en ganado caprino: vigorización energética (flushing) en hembras reproductoras y uso de Calliandra calothysus en cabros destetados. Tesis de Maestría. San Juan: Universidad de Puerto Rico. 76 p.

2. Aguilar-Pérez C, Ku-Vera J, Centurión-Castro F, Garnsworthy PC. 2009. Energy balance, milk production and reproduction in grazing crossbred cows in the tropics with and without cereal supplementation. Livestock Sci 122: 227-233. doi: 10.1016/j.livsci.2008.09.004

3. Aliaga L, Rodríguez $H$, Borja $A$, Núñez E. 1984. Sistema de empadre con flushing en cuyes. En: VII Reunión APPA. Lima. Asociación Peruana de Producción Animal.

4. Aliaga L, Moncayo R, Rico E, Caycedo A. 2009. Producción de cuyes. Lima: Fondo Editorial de la Universidad Católica Sedes Sapientiae. 808 p.

5. Ara M, Jiménez R, Huamán A, Carcelén, Díaz D. 2012. Desarrollo de un índice de condición corporal en cuyes: relaciones entre condición corporal y estimados cuantitativos de grasa corporal. Rev Inv Vet Perú 23: 420-428. doi: 10.15381/rivep.v23i4.948

6. Barrios-De-Tomasi J, Barrios-DeTomasi E, Vergara-Galicia J. 2013. Efecto de la obesidad en la reproducción femenina. Rev Mex Cienc Farm 44: 448-466.

7. Brewer CJ, Balen AH. 2010. The adverse effects of obesity on conception and implantation. Reproduction 140:347364. doi: 10.1530/REP-09-0568

8. Bustamante J. 1993. Producción de cuyes. Lima: Facultad de Medicina Veterinaria, Universidad Nacional Mayor de San Marcos. 259 p.

9. Cáceres F, Jiménez R, Ara M, Huamán HA, Huamán AE. 2004. Evaluación del espacio vital de cuyes 
criados en pozas. Rev Inv Vet Perú 15: 100-112. doi: 10.15381/rivep.v15i2.1577

10. Chauca L. 1997. Producción de cuyes (Cavia porcellus). Roma: FAO. 77 p.

11. Church DC, Pond WG, Pond KR. 2010. Fundamentos de nutrición y alimentación de animales. México: Limusa Wiley. $635 \mathrm{p}$.

12. Forcada F, Abecia A, Zarazaga L, Lozano J. 1992. Influencia del plano de alimentación sobre los parámetros reproductivos en ovejas de reducido nivel ovulatorio. Arch Zootéc 41: 113-120.

13. Hashem NM, El-Zarkouny SZ. 2014. Effect of short-term supplementation with rumen-protected fat during the late luteal phase on reproduction and metabolism of ewes. J Anim Physiol Anim Nutr 98: 65-71.doi: 10.1111/jpn.12032

14. Jiménez R. 2007. Uso de insumos agrícolas locales en la alimentación de cuyes en valles interandinos. Arch Latinoam Prod Anim 15: 229-232.

15. Jiménez R, Huamán R. 2010. Manual para el manejo de reproductores híbridos especializados en producción de carne. El Mantaro, Perú: INCAGRO ACRICUCEN- UNMSM. 175 p.

16. Laforé M. San Martín F, Bojórquez C, Arbaiza T, Cárcelen F. 1999. Diagnóstico alimenticio y composición químico nutricional de los principales insumos de uso pecuario del valle del Mantaro. Rev Inv Vet Perú 10(2): 74-78.

17. Martínez de Acuero M, Mazzarri G, Rodríguez J, Quintana H, Chicco C. 1986. Suplementación energética y proteica pre-servicio en ovejas West African. Zootecnia Trop 4(1-2): 19-28.
18. Martínez de Acurero M. 2000. Estrategias de suplementación en ovejas. Centro de Investigaciones Agropecuarias del Estado de Zulia. FONAIAP Divulga N. ${ }^{\circ}$ 66. [Internet]. Disponible en: http:// sian.inia.gob.ve/repositorio/revistas_tec/ FonaiapDivulga/fd66/texto/ estrategias.htm

19. Michael CL, Bonnet X. 2011. Influence of body condition on reproductive output in the guinea pig. J Exp Zool A Ecol Genet Physiol 317: 24-31. doi: 10.1002/jez.714

20. Pérez-Pérez A, Sánchez-Jiménez F, Maymó J, Dueñas JL, Varone $C$, Sánchez-Margalet $V$. 2015. Role of leptin in female reproduction. Clin Chem Lab Med 53: 15-28. doi: 10.1515/cclm2014-0387

21. Rico NE, Azuga SM, Holting G. 1994. Alimentación en cuyes. La Paz, Bolivia: Proyecto de mejoramiento genético y manejo del cuy en Bolivia (Mejocuy). Universidad Mayor de San Simón. Boletín Técnico $\mathrm{N}^{\circ} 1$.

22. SAS Institute. 1990. User's Guide, v. 6. $4^{\text {th }}$ ed. Cary, NC: SAS Institute Inc. $943 \mathrm{p}$.

23. Smith GD, Jackson LM, Foster DL. 2002. Leptin regulation of reproductive function and fertility. Theriogenology 57 : 73-86. doi: 10.1016/S0093-691X(01) 00658-6

24. Zaldivar M, Chauca L, Muscari L. 1988. Características básicas del cuy. Santiago de Chile: Oficina Regional de la FAO para América Latina y el Caribe, Organización de la Naciones Unidas para la Agricultura y la Alimentación. $\mathrm{p}$ 20-37. 